\title{
CHRONIC NEPHRITIS.
}

\author{
By A. G. GIBSON, M.D., F.R.C.P. \\ (Physician to the Radcliffe Infirmary, Oxford.)
}

\section{I.-Chronic Parenchymatous Nephritis.}

Etiology. The disease is most frequently seen in children and young adults. It may follow general infective diseases such as scarlet fever, erysipelas and septic sore throat, and is seen in those with large tonsils or some other septic focus in the naso-pharynx. A chill may precipitate the first symptom of œdema.

Morbid Anatomy. The kidney is large, pale and cream coloured; the surface is smooth and the capsule which is thin strips easily. Injected veins may be seen on the surface. On section the cortex is swollen and yellowish white, the pyramids are congested. Examination under a lens may show that some of the glomeruli are atrophied and whitish, others may show hæmorrhages; the convoluted tubules are prominent, cellular and fatty. Interstitial changes are always present and best shown up by special microscopic stains. In cases which have lasted longer the small white kidney is seen. It is pale, the surface is granular and on section the cortex is much reduced with the deposition of fibrous tissue. There is usually fatty degeneration of the heart; hypertrophy may be present but is not a marked feature except in cases that have lasted a long time; dilatation of the cavities, shown by the increased diameter of the auriculo-ventricular rings may have been a terminal event. The main feature post mortem, however, in these cases is an enormous odema of the whole body. All the loose tissues are filled with extra fluid and clear fluid is present in the pericardial, pleural, peritoneal and cranial cavities.

Clinical Features. The onset is often insidious and the initial puffiness of the eyes may be noticed by the patient's friends. It may, however, come on suddenly or follow an attack of acute nephritis. Other symptoms are dyspepsia, loss of energy, or a feeling of ill health. The odema is earliest shown in a puffiness under the eyes and is more marked in the morning than in the evening. If the patient is up and about the œdema is usually present in the feet and legs, and may simulate cardiac œdema, but there is always a little puffiness under the eyes, and the " unshed tear" of the conjunctiva is another indication. As the cedema progresses the patient becomes swollen and pale and odema may be found in the body cavities. In the fully marked case we have the "large white man with the large white kidney." Loss of appetite, nausea and vomiting may be present. Diarrhoea is not usual but may be a terminal event. The tongue is furred and the breath foul. Anæmia and wasting are constant but the latter may be masked by the œdema. In the early stages at least, pyrexia of moderate degree is present.

Urine. The amount of urine passed per diem is less than normal. The specific gravity is higher than normal and it is turbid with urates or smoky from blood. The quantity varies with the amount of œdema and increases with any improvement or treatment directed towards getting rid of the œedema. Albumin is present in large amount; all varieties of casts may be seen especially epithelial and fatty; leucocytes are often numerous and there may also be red cells. The chlorides of the urine are diminished. Renal efficiency tests and blood urea, however, are often normal. 
In severe cases the fat in the blood is increased and when drawn the serum that settles may be turbid. Sometimes also, the effusions are turbid from this cause (pseudochylous).

Prognosis. This is always grave and uncertain. Occasionally a patient recovers to apparent good health after several months of illness only to relapse again from a trivial infection. The majority of patients show no marked improvement with treatment and ultimately die. But sometimes, after a year or more, the patient may take a turn for the better and recover completely.

Death is brought on by cardiac failure, some infection such as pneumonia, pericarditis, or by the gradual failure of renal function.

Treatment. Patients are best treated in bed, though if he finds himself more comfortable sitting up, this may be allowed. The generalized oedema requires care of the skin and particularly of the pressure points. The best position in bed is usually that of being propped up by pillows in that it allows the odema fluid to gravitate to the lower part of the body. If the patient is not ill nourished a trial may be made from time to time, say once a week or once a fortnight, of twenty-four hours on orange juice only, as is used in acute nephritis; but as these patients are often very ill nourished such a diet must be used sparingly. Any diet must ensure that the patient is well nourished, for the loss of albumin by the kidney is a considerable drain. The most useful is Epstein's diet which rests on high protein, a minimum of salt and reduction in the fat intake. The protein may be given up to $240-250$ grammes, fat up to 40 grammes and carbohydrates up to 300 grammes, and the fluid intake should be lessened to not more than I,500 cc. (3 pints). The good results of this diet appear to be the result of the increased diuretic action of urea in the blood. This action may be produced by the administration of urea by the mouth, 30 grammes daily. Hot air baths, by relieving the kidney for the time being, often exert a beneficial effect. They are best given by a cradle attached to the inside of which there are several incandescent lamps. The pulse rate is taken every 5 or Io minutes and the patient's comfort or distress should be noted. Twenty minutes to half an hour is the average length of time for the bath, and it may be repeated twice or three times a week, or daily, if the patient is not too exhausted. It is useless to continue these baths if the patient gets no benefit. The best indication of benefit is sweating during the bath, followed by an increase in the quantity of urine.

One factor in the persistence of chronic parenchymatous nephritis may be a focus of infection. It is not wise to deal with this until a remission occurs, but it may be possible by simple measures such as the toilet of the throat to lessen the burden of the infection acting on the kidney. Occasionally a trial of protein shock in mild degree is of benefit. Cautious trial of salyrgan or one of the mercurial diuretics would be justified in obstinate cases.

\section{II.-Chronic Interstitial Nephritis.}

\section{(Azotæmic nephritis, red granulax contracted kidney, general arteriosclerotic kidney).}

Etiology. This variety of nephritis seldom occurs before the age of 40 and is commoner in males. It has a tendency to affect certain families in whom arterial degeneration occurs early. Overwork, overeating, over-indulgence in alcohol, syphilis, gout and lead are causative factors. Odd cases are now and then met with in younger persons and even children. Inherited syphilis may at times be the cause in these patients. 
Morbid Anatomy. The kidneys are small and red, and on stripping the capsule the surface is granular without adhesions. On section the cortex is diminished by half or two-thirds, the colour is deep red, there is no indication of fatty degeneration of the tubules, the substance of the kidney is tough from fibrous tissue and the vessels are prominent. Microscopically the glomeruli are atrophied, their capsules are thickened and the remains of the tubules are dilated with some degenerative change in the cells. The arteries are thickened, especially the intima, and in those that are fully patent the muscle is hypertrophied. Other organs affected are the heart which is constantly hypertrophied, concentrically at first, but with dilatation subsequently. The hypertrophy especially affects the left ventricle though both sides are implicated. The arteries in other parts of the body such as the base of the brain are thickened.

Pathogenesis. It is not possible to give a clear picture of the origin of the condition without postulating the substances in the blood that have an irritating effect on the kidneys. Hyperpiesis alone appears not to be able to give rise to this form of renal disease. The toxins of gout, syphilis and lead must be constantly kept in mind as the damaging factors in those cases in which they are present. It is doubtful if focal sepsis has anything to do with the evolution of the disease. The hypertrophy of the heart is to be explained solely on the hyperpiesis which is the normal circulatory reaction to this form of renal damage.

Symptoms. The onset is insidious and the disease may have lasted several years before any symptom attracts the patient's attention, or it may be found accidentally in a routine examination for life assurance, or the full course of the disease may be gone through until a fatal cerebral hæmorrhage terminates the patient's life.

The patient becomes aware of his condition by alteration in appetite, distaste for food, dyspepsia and even nausea, or he may suffer from periodical headaches and giddiness. There is general weakness and loss of the normal power of work. A good example of this may be found in the story of Lady Anne Conway (Conway Letters I642-I684, Ed. M. H. Nicolson, London I930). When the heart shows signs of insufficiency, breathlessness and palpitation may be the most noticeable symptoms. Nocturnal micturition with the passage of large quantities of dilute urine may cause the patient to seek advice. The sight may fail owing to changes in the retina.

The urine is increased markedly in amount and there is frequency, especially evident at night. The urine is pale and clear and the specific gravity is persistently low. Albumin if present is in very small amount. The amount of urea is low and the chlorides are normal. In the centrifugalized deposit a few casts, hyaline or granular, may be found. Occasionally there may be hæmaturia, and, if cardiac failure comes on, the quantity of urine diminishes without any alteration in the specific gravity. The albumin in these circumstances increases.

In the circulatory system the heart shows signs of hypertrophy in a displaced apex beat, increased cardiac dullness and increase of the cardiac shadow, especially to the left, under X-rays. In dilatation there may be a high pitched systolic murmur at the apex. At the aortic area the second sound is greatly accentuated. In those cases in which cardiac failure comes on the heart enlarges; the apex beat may be found as far out as the mid axillary line.

Amongst respiratory symptoms emphysema commonly interferes with the delimitation of the heart. The chest is full and emphysematous cushions may be 
seen above the clavicles on coughing. The patient has a tendency to suffer from intercurrent infections; bronchitis, pneumonia or pleurisy are common complications. Alterations in respiration such as attacks of dyspnoea may be cardiac or uræmic. Cheyne-Stokes breathing may occur when the blood pressure is diminished towards the end. Sudden œedema of the glottis or of the lungs is sometimes seen.

Amongst digestive symptoms dyspepsia, nausea and loss of appetite are constantly present, more marked in the morning. The tongue is furred and the breath often has a characteristic uræmic smell. Uræmic vomiting may be a difficult symptom to counteract. Constipation is usual in the early stages but later on may be replaced by diarrhœa often with the passage of blood and a picture of ulcerative colitis.

Of nervous symptoms headache is the earliest and the most constant. It is. often mistaken for migraine in that it is paroxysmal. Attacks of giddiness, loss of consciousness, twitchings and neuralgia also occur. Any form of psychical abnormality may be present from irritability and great mental asthenia to delusional insanity. Amongst eye symptoms failing vision is frequent. This may be transient or permanent. Transient amaurosis is often due to spasm and retinal changes may be absent. Permanent interference with vision may be associated with albuminuric retinitis and optic neuritis. In the former there may be scattered cotton wool patches in various parts of the retina and in the course of the vessels hæmorrhages of various ages. It is essential to look round the fovea for the starlike pattern of this form of retinitis.

Edema is not a marked feature of the condition though the "unshed tear" may be present and the feet may show a tendency to swell towards the evening. The skin is consistently dry, slightly roughened, and the complexion muddy. In the late stages a powdery efflorescence may appear in certain parts. Nose bleed and other hæmorrhages such as conjunctival, retinal or renal may be present. When the hæmorrhage is profuse as in nose bleed the patient often feels a sense of relief. Small cerebral hæmorrhages may give rise to transient giddiness. In the late stages there is always a slight secondary anæmia which is noticed by the patient's friends. The blood urea is usually above normal and increases in the late stages. It may, however, be diminished by dieting or other treatment.

Diagnosis. This disease has to be distinguished from large prostate with frequency of micturition and from diabetes with the passage of large amounts of urine. In the former, however, the specific gravity is not diminished and there may be pyuria, while in the latter the presence of sugar would be noted. Special care should be taken, when symptoms of indefinite indigestion persist, not to be satisfied with the diagnosis of gastritis. The œdema of cardiac disease may simulate that of renal disease, especially if there is uræmic breathlessness. The two conditions indeed may exist together. A chronic bronchitis, especially if it shows asthmatic paroxysms, may simulate respiratory symptoms in chronic interstitial nephritis. Cerebral tumour may be suggested by headache, vomiting and changes in the optic disc.

Course and Prognosis. The disease is consistent with fair, even vigorous, health for many years. Intercurrent infections occur from time to time. Renal retinitis is of particularly grave significance and the length of life following this complication is seldom more than two years. The blood urea or renal efficiency tests enable the progress of the nephritis to be assessed from time to time. The patient dies from uræmia, cerebral hæmorrhage, cardiac failure, intercurrent infections or acute conditions of the kidney. 
Treatment. The aim of treatment is to delay a process which once started $\frac{3}{2}$ cannot be completely removed. Two ætiological factors can be controlled, namely,.. lead and syphilis. All sources of poisoning through lead should be removed, and $\vec{F}$ syphilis should be vigorously treated by the newer remedies as well as by mercury $\frac{0}{0}$ and potassium iodide. A gouty person should avoid such meat and drink as brings on the attack, and in general the patient should regulate his life so as to avoid all excess - mental, physical, dietetic, and especially as regards alcohol. With care a patient known to have this disease may be steered through a reasonable span of life and may take his part in business or affairs. Exercise should not be banned, in fact a moderate amount of exercise tends to keep the peripheral circulation active, but it is better to restrict exercise to more moderate exertion, such as golf and tennis and walking. The diet should be brought down to physiological limits, but apart from meat it need not be unduly restricted, and the effect of all 8 treatment should be gauged by a regular record of the weight and the condition of the urine. Any undue restrictions should be avoided. The fluid intake should not $\vec{\omega}$ be restricted except in the case of alcohol which is best avoided altogether, unless $\vec{\omega}$ in the case of older patients who have been accustomed to a daily ration. The of bowels should be kept active and an occasional mercurial pill or morning saline $A$ may be given. The raised blood pressure may of itself produce symptoms that 9 annoy the patient. This is true of headache, which, apart from the general methods $\vec{T}$ just detailed, may be relieved by rest and venesection. The latter is often done with $\stackrel{\mathbb{D}}{\circ}$ benefit every three months, withdrawing $\mathrm{I}$ to $\mathrm{I} \frac{1}{2}$ pints. The value of vaso-dilators is doubtful, but they are harmless and in difficult cases may be given a trial. 产 Liquor trinitrini up to 3 minims twice daily or erythrol tetranitrate $\frac{1}{2}$ to I grai $\vec{c}$ three times a day are the best drugs.

Individual symptoms should be treated as they arise. Cardiac failure requires full digitalization and the other methods for that condition. Uræmia should be treated by hot air baths and saline purgatives. Opium in some form is necessary for restlessness and delirium as well as for dyspnœa and Cheyne-Stokes respiration. Chloroform may be necessary to control convulsions which may also be treated by venesection and intravenous infusion.

\section{III.-Chronic Focal Nephritis.}

Under this heading three varieties can be distinguished.

I. Chronic Glomerular Focal Nephritis (which is part of the disease infective endocarditis).

Morbid Anatomy. In an early case the kidney is known as the flea bitten kidney. It is not enlarged and is slightly paler than normal; on section the cortex is not diminished and here and there may be seen small petechial hæmorrhages. which are the site of minute emboli in the glomerular arteries. The condition is best seen on stripping the capsule where the petechial hæmorrhages may be few or many. In the later stages fibrosis causes contraction of the kidney, diminution of N the cortex and a generalized dimpling of the surface.

Symptoms and Course. Blood can be detected microscopically in the deposit of the urine together with a trace of albumin. In that it is a focal lesion there are seldom any symptoms attaching to the kidney. Occasionally, however, there may

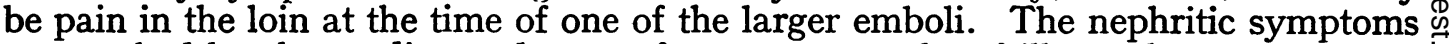
are masked by the cardiac and even after many months of illness there may be no 0 signs of deficiency of the kidney. If the patient survives the cardiac lesion long 0 enough there may ultimately ensue the symptoms of chronic parenchymatous 
nephritis with œdema, headache, vomiting, pallor and the symptoms of chronic uræmia, and cases are recorded in which the patient has died from renal rather than from cardiac complications.

\section{Focal Arteriosclerotic Nephritis.}

Morbid Anatomy. This is the true senile arteriosclerotic, atheromatous or dimpled kidney. The kidney is small and dark red. On section the cortex is diminished in particular areas with the deposition of some fibrous tissue. On stripping the capsule there are local depressions where the cortex has become atrophied, the result of arterial blockage through atheroma. The arteries everywhere can be seen to be thickened.

Symptoms. These are trivial and inconspicuous. The patient, who is in the degenerative period of life, may complain of finding that his urine contains blood. $\mathrm{He}$ is afebrile, shows no other sign of renal disease, the urine contains a small amount of albumin, numerous blood cells and occasionally blood or cellular casts. The patient suffers no inconvenience though slight albuminuria may remain for a time. This condition, which simulates nephritis as regards the urine, may recur, but it is entirely benign and may never produce any renal insufficiency. It is possible that the occasional presence of albumin in the urine of old persons may be attributed to local arterial ischæmia.

\section{Chronic Focal Pyelonephritis.}

On the one hand we have the pyæmic kidney with multiple septic infarcts and, on the other, an occasional focal sepsis of the kidney from some chronic distant lesion. The clinical symptoms and signs are not easy to distinguish from pyelitis, if indeed, such distinction is possible. In focal sepsis of the kidney, however, albuminuria is present whereas in pyelitis it is trivial or absent. Not infrequently a case which in the early stages shows pus in the urine may go on to a condition in which there are casts (cellular and granular) and which is indistinguishable from parenchymatous nephritis.

Morbid Anatomy. The kidney in the early stages shows triangular areas of the infarct type, sometimes necrotic, sometimes septic, but always inflammatory. The healing of these lesions produces atrophy of the kidney substance according to the initial size of the patch. Occasionally small or large cysts may result. The rest of the kidney shows little alteration from the normal and it is this contrast between the scarred and the normal kidney tissue that proclaims its origin. In the late stages the kidney is atrophic from the gradual destruction of kidney substance and constitutes one variety of the small white kidney.

Symptoms. During the main course of the illness there may be no symptoms. The patient, however, suffers from albuminuria, worse at times than at others, and the urine contains pus from time to time. The chief symptoms are shown when the amount of kidney substance destroyed interferes with the kidney reserve and chronic uræmia develops. There is seldom any hypertrophy of the heart or true retinitis, though there may be hæmorrhages in the eyes or elsewhere.

Treatment. All focal sepsis that is possible should be eliminated and the general management of the case should be on the lines of chronic interstitial nephritis. 